\title{
Analytical and Numerical Model for Evolution of Minimal Cell with Infinite Number of Energy Links
}

\author{
Moldavanov A.V. \\ 2774 Sunnybridge Drive, Burnaby, British Columbia, Canada \\ $\underline{\text { trandrei8@gmail.com }}$
}

\begin{abstract}
Analytical simulation of energy evolution of minimal cell as an open thermodynamic system is conducted based on the mathematical model utilizing formalism of randomized energy continuity equation. Approximation of open system is achieved by use of infinite number of links with environment in assumption that all links are executed by energy continuity equation. As a result, this approach deals with the phase space with determined exchange rate and random interface factor. Found solution is function of a system energy interface and in its physical meaning is an integral efficiency of energy exchange. Picking up relations from an evolutionary geometry, we clarify meaning of obtained results for energy development of cell. Found solution possesses the built-in mechanism for phasing, dynamic transformation of an energy exchange's structure, and predetermined change of range for random variations. For verification, the basic results are duplicated by numerical methods.
\end{abstract}

Key words: evolution, randomizing, phasing, decomposition, minimal cell.

\section{Математическое и численное моделирование энергетической эволюции простейшей клетки с инфинитным числом связей}

\author{
Молдаванов А.B. \\ 2774 Sunnybridge Drive, Бёрнаби, Британская Колумбия, Канада
}

\begin{abstract}
Аналитическое моделирование эволюции простейшей клетки как открытой термодинамической системы выполняется на основе математической модели рандомизированного уравнения непрерывности энергии. Приближение открытой системы достигается использованием неограниченного числа связей с внешним окружением в допущении, что каждая связь работает на основе формализма уравнения непрерывности. В этом смысле, предлагаемая модель использует фазовое пространство с детерминированной интенсивностью энергообмена и случайным интерфейс-фактором. Найденное решение представляет собой немонотонно меняющуюся функцию энергетического интерфейса системы и, по своему физическому смыслу, является интегральной эффективностью энергообмена. Использование последних результатов в эволюционной геометрии позволяет дать корректную интерпретацию найденным математическим соотношениям. Полученное решение характеризуется наличием встроенных механизмов фазирования, динамического преобразования структуры энергообмена и упорядоченного изменения величины случайных вариаций. Для проверки точности полученных результатов основные соотношения были продублированы с помощью вычислительных методов.
\end{abstract}

Ключевые слова: эволюччия, рандомизация, фазирование, декомпозиция, простейшая клетка.

\section{Introduction}

These days, there exists a general consensus that in its simplest manifestation life comes in the form of some kind of cell $[1,2]$. In other words, we are forced to accept that a model of minimum life should be that of a minimal cell $(M C)$. And suggest that such cell-like compartments have capacity to evolve.

Also, it looks naturally if we think of a $M C$ from physical point of view as an open thermodynamic system (OTS) capable of self-maintenance due to a process of permanent energy exchange using available chemical infrastructure within some boundary [3]. And evolution to advanced living beings as autonomous systems with open-ended evolution capacities must include an energy transduction apparatus in its simplest form coming as the set of multidirectional energy currencies.

Thus, research of the evolution potential for the celllike compartments that have just the very basic 
fundamental attributes of life (presumably wellorganized biopolymeric assemblies) [4] can provide better understanding for an essence of energy evolution.

Study of $M C$ based on the mathematical model of the system with infinite number of connections with external environment through energy continuity equation $(E C E)$ was performed in [5]. Investigation of topology relations within evolutionary triangles was conducted in [6]. In this report, we combine results [57] in application to an energy development of $M C$.

\section{Model}

\subsection{Assumptions}

It is assumed a uniform probability distribution for all used random quantities, and an infinite number of independent links between $M C$ and external environment which is represented by a thermal bath of unlimited capacity. Random energy flows $\mathbf{J}_{\text {in }}$ (inward $M C$ ) and $\mathbf{J}_{\text {out }}$ (outward $M C$ ) cannot coexist at the same time, i.e. general energy flow $J=J_{\text {in }} \oplus J_{\text {out }}$, where $\oplus$ denotes exclusive disjunction.

\subsection{Approach substatntiation}

Energy conservation law in its differential form (energy continuity equation $(E C E)$ )

$$
\frac{\partial \varepsilon}{\partial t}=-\operatorname{Div} \mathbf{J}
$$

is one the most fundamental relations, here $\varepsilon$ is an energy volume density, $t$ is time, and Div is a divergence operator. $E C E$ is of the universal nature and it deals with the interface between $M C$ and energy flux by default.

We assume that flux $\boldsymbol{J}$ and density $\varepsilon$ are not attributed to any specific physical process. As soon as some energy transportation occurs contributing to the change of $M C$ energy balance upon arrival, it falls under category for our consideration.

\subsection{Mathematical formalism}

Based on (1), introduce infinite system of $E C E$

$$
\left\{\begin{array}{c}
\frac{\partial \varepsilon_{1}}{\partial t}=-\operatorname{Div} \mathbf{J}_{1} \\
\frac{\partial \varepsilon_{2}}{\partial t}=-\operatorname{Div} \mathbf{J}_{2} \\
\cdots
\end{array}\right.
$$

System (2) can be converted to randomized ECE (RECE)

$$
\frac{d U}{Q}=-\frac{d J}{J} x
$$

where $x=\cos \varphi, \varphi$ is an angle between the direction of an instantaneous change of $J$ and the unit normal, $d U=\varepsilon \cdot d V, Q=J \cdot d S d t[7]$.
Solution of (3) for function of an energy interface

$$
\Upsilon=\iint_{M} \frac{d U}{Q}=-\iint_{M} \frac{d y}{y} x
$$

is

$$
\Upsilon(y)=y-y \ln y
$$

which is shown in Fig. 1, where $y$ (energy exchange rate) $=J / J_{0}$, normalizing constant $J_{0}>0, M \subseteq \mathbf{R}^{2}$ is the phase space for all possible states of $\delta \Upsilon$.

Appropriate solution for entropy $S$ is

$$
\Delta S=\ln |\ln y|+H_{x}
$$

which is shown in Fig. 2, where $H_{x}$ is entropy probability distribution for $x$ on the compact support $[-1,1]$.

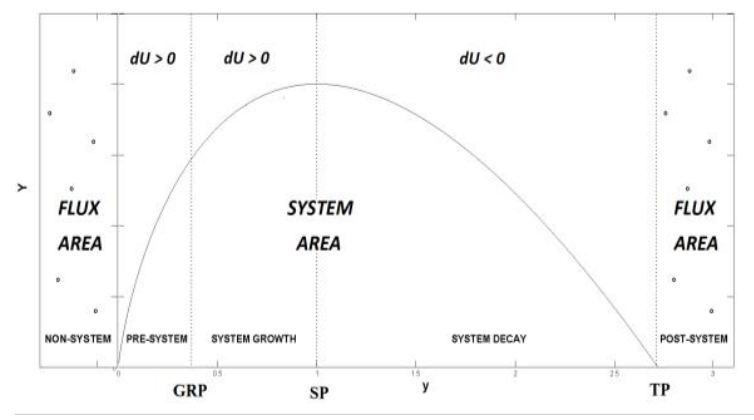

Fig. 1. Dependence of integral efficiency $\Upsilon$ (vertical axis) on rate of energy exchange between $M C$ and its surroundings $y$ (horizontal axis). Two major zones are shown - the "Flux Area" and the "System Area". In the "Flux Area", there is no signatures of OTS while in the "System Area" it is. The phase transition points $G R P(y=1 / e), S P(y=1), T P(y=e)$ are also shown, where $e$ is Euler number. Area with $d U>0$ designates $y$-range where in average the growth of internal energy $U$ is observed, whereas $d U<0$ means an opposite case.

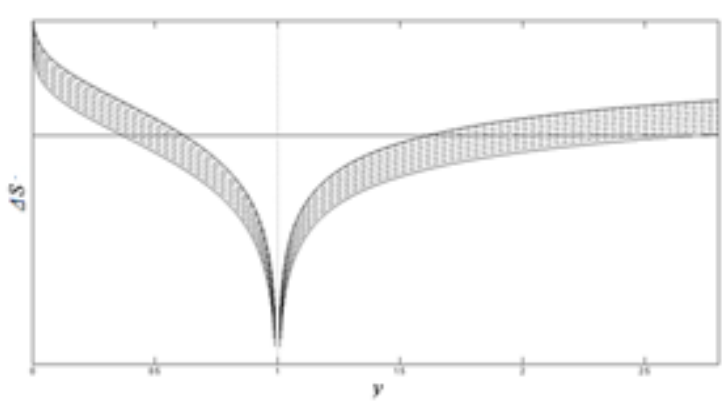

Fig. 2. Dependence of entropy $\Delta S$ (vertical axis) on rate of energy exchange $y$ (horizontal axis). The range for $y$-roots of entropy $\Delta S$ is in between the pairs of first and second harmonic of the discrete spectrum for evolving $M C$. Plot demonstrates an asymmetry relative to the line $y=1$.

\subsection{Singularity of solution at point $y=1 / e$}

Our research showed that in the range $0 \leq y \leq 1 / e$ the outputs favouring to flux $y=y_{\text {in }}$ (inward $M C$ ) are more probable than to flux $y=y_{\text {out }}$ (outward $M C$ ), whereas 
beyond point $y=1 / e$ (in the range $1 / e \leq y \leq e$ ) situation changes to opposite, here $e$ is Euler number. The same scenario happens with the entropy rate $d S / d y$, which grows at $y<1 / e$ and decreases at $y>1 / e$. Similar behaviour exists for the amplitude of fluctuations of $d S / d y$ either, and quantity $d S / d y$ demonstrates maximum at $y=1 / e$. Besides, as is shown in 2.5 , at $y=1 / e$ spectrum of solution changes from the continuous to the discrete. Another thing is that at $y=1 / e$ a leakage of microstates to a complex domain commences. Moreover, amplitude of $x$-fluctuations starts to decrease. And, finally, $y=1 / e$ is one of the roots of entropy (6).

Above results support position [8] that system's native ability to adapt to changes in environment goes through suppressing big fluctuations around critical points, dissipating abundant energy ( $y_{\text {out }}$ in this model) and reflect the well-known property of OTS to operate close to the phase transition point keeping away from equilibrium [9].

\subsection{Discreteness of energy spectrum}

From (5), to meet the suitable boundary conditions should hold

$$
1= \pm n \ln y_{n},
$$

as a result, at $|\ln y| \leq 1$, i.e. in the range $[1 / e, e]$

$$
y_{n}=\exp \left[ \pm \frac{1}{n}\right]
$$

So, the $y$-points (8) are the nodes of the discrete spectrum and the point $y=1 / e$ is the first node, where energy spectrum changes from continuous to discrete.

\subsection{Connection between physical and topological parameters of the model}

It is possible to show that the thermodynamic and the topological parameters in considering model are linked through the factor $k$

$$
\kappa=1-\operatorname{sign}(\ln y)|\ln y|
$$

which is an eigenvector for an energy interface operator (5), where sign is the sign function [10]. Above provides grounds for direct applying of relationships originally developed in evolutionary geometry [6] to energy ratios (5-8).

\subsection{Evolutionary topology of energy exchange}

Based on results [6], for the evolutionary triangles defined by legs $y$ and $\Upsilon$ (Fig. 3), there exists relation between triangle's areas

$$
\left(\frac{2 S_{n}^{T}}{S_{n}^{C}}\right)^{p}=\gamma(k)\left(\frac{S_{n}^{F}}{2 S_{n}^{T}}\right)^{p}=\tau,
$$

where $\gamma(k)=\left(4 / k^{2}\right)^{p}, p= \pm 1, n=1,2, \ldots$.

In its physical meaning, area of any triangle shown in Fig. 3 and shown in (10) is the portion of the product $y \Upsilon$. The latter is the weighted efficiency of energy exchange between external environment and $M C$. Then, $S^{T}$ (transfer triangle), $S^{C}$ (core triangle) and $S^{F}$ (full triangle) $=2 S^{T}+S^{C}$, also refer to the portions of this weighted efficiency.

From (10) follows

$$
\eta^{2}+2 \eta-k^{2}=0
$$

where

$$
\eta=\frac{S^{C}}{S^{T}}=\sqrt{k^{2}+1}-1
$$

which provides breakdown for evolutionary triangle at any node $n$. As such, (11) deals with the predetermination in appearance of the structure for energy exchange (fine structure of spectral harmonic) as to survive, $y$-mode should have maximum possible value of $1 / \eta$. Hence, evolution manifests by a mixture of the quickly depressing modes at $y \neq y_{n}$ which do not have maximum $1 / \eta$ and the stable modes at $y=y_{n}$ when $1 / \eta$ is of its maximum (shown in Fig. 4). In this sense, we could say on existence of evolution infrastructure in the energy development of $M C$. Of course, use of this infrastructure is subject of attainment of suitable energy exchange rate $y$ which is not assured.

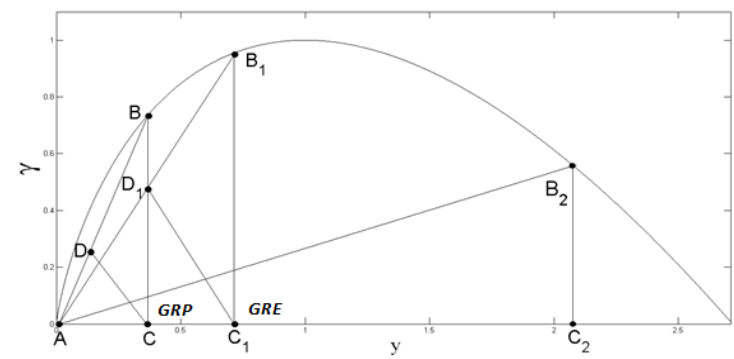

Fig. 3. Evolutionary triangles formed by curve of integral efficiency $\Upsilon$. Designation of the axes is the same as in the Fig. 1. $\triangle A B C$ is defined by leg $y=A C$ and $\Upsilon=B C$ which fits to the state of dynamic balance between, on the one hand, the area $2 S^{T}=2 \triangle A C D$, and on the other hand, the area of $S^{C}=\triangle B C D$. It is seen that evolutionary triangles are not similar; instead, they are obeyed to equation of $M C$ evolution (11) claiming predetermined character of changes.

At $y=1 / e$, value $\tau$ in (10) exactly equals to a golden ratio, so an instantaneous internal structure of an energy exchange in the $M C$ achieves the state of dynamic balance [11]. That is why further we will call the point $y=1 / e$ as Golden Ratio Point (GRP) (shown in Fig. 3).

\section{Results}

\subsection{Built-in phasing of evolution}

It is clear that GRP separates two physically dissimilar $y$-ranges when evolving $M C$ makes qualitative transition from one operational mode to another. We can call these two ranges as agenesis $(y<G R P)$ and genesis $(y>G R P)$. It is worth to note 
that in this model genesis is different from agenesis, first of all, by emergence of new qualities in functioning of $M C$ that were not presented before. It is quite aligned
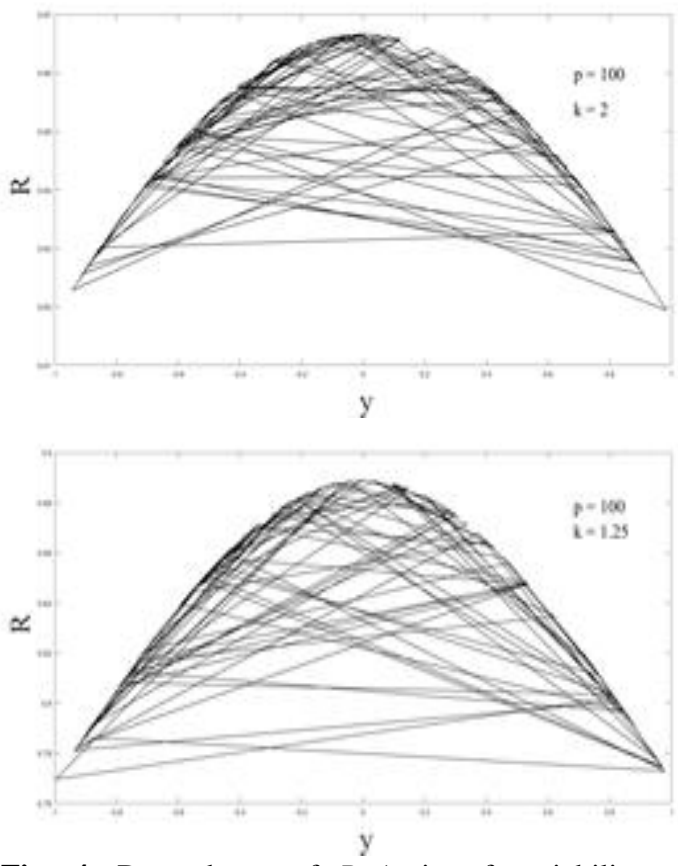

Fig. 4. Dependence of $R$ (ratio of variability to inheritance) on the random step of evolution at fixed $k$. Shown two cases for volume of random steps $p=100, k=1.25$ (lower panel) and $p=100, k=2$ (upper panel). It is seen that maximum $R$ demonstrate spectral modes with the maximum ratio of variability to inheritance (corresponds to zero deviation from the position of the stable mode $y_{n}$ ). Numerical simulation was done in the MATLAB environment.

with [12] where is stressed that emergence of quality difference for evolving entity is identifiable not by the new parts but novelty of operation.

However, GRP is not unique. Other nodes of the discrete spectrum can demonstrate the phase transition properties either [7].

So, evolution of $M C$ could be considered as succession of the qualitative jumps between unlimited counted number of stable spectral harmonics (evolution infrastructure), where each range in between the adjacent harmonics is a separate stage of evolution. In this sense we may say about a phasing of $M C$ evolution.

\subsection{Dynamic decomposition of structure for energy exchange} ratio:

Based on (10), it is possible to obtain the following

$$
\frac{S_{n+1}^{C}}{S_{n}^{T}}=k^{2} \frac{S_{n}^{T}}{S_{n}^{C}}
$$

It shows that $S^{C}$ is the nodal quantity which keeps its value (area) for each node $n$ (parent/offspring) at passing to / from another node $n+1$ (offspring/parent). However, $S^{T}$ is the different kind of quantity which supports trading and is related to the transfer process.
Coupling between $S^{C}$ and $S^{T}$ makes evolutionary triangle to go further in its development.

As mentioned, $S^{C}$ and $S^{T}$ are the portions of the weighted efficiency of energy exchange, so it is logically to associate $S^{C}$ with the mechanism of energy inheritance while $S^{T}$ with the that of energy variability.

Then, returning to the sect. 2.7, at GRP inheritance and variability is in the state of a dynamic balance.

\subsection{Energy covariant reduplication}

As fairly noted in [13], a quantitative understanding of evolution would flesh out the balance between evolvability and robustness $\left(S^{T}\right.$ and $S^{C}$ in this model). Energy evolution of $M C$ (11) is governed by $\eta$ (12). Therefore, to evolve $M C$ should support regular change of the random $S^{T}$. In this sense, during evolution, $S^{T}$ should experience regular alternations either. In analogy to phenomenon of covariant reduplication [14], it can be called as an energy covariant reduplication.

\subsection{Driving force of $M C$ energy evolution}

For this model was proved that

$$
\frac{\delta \Upsilon}{d t} \leq 0
$$

[5]. Then, at completion of each random loop, function $\Upsilon$ experiences the non-zero change $\delta \Upsilon$. It means that description of $M C$ acquires one more degree of freedom thereby becoming the two-dimensional one which brings asymmetry for random outcomes leading to

$$
P(\Delta y>0) \geq P(\Delta y<0)
$$

where $\Delta y$ is a random step in $M C$ evolution, $P$ is probability. The results of numerical simulation of above asymmetry are shown in Fig. 5.

So, we see that (15) creates general probabilistic background which makes complexification and evolution of $M C$ at some conditions unavoidable. Author [15] comes to close conclusion stating that the adaptation feature of systems "... may be embedded more deeply in the thermodynamics of complex systems".

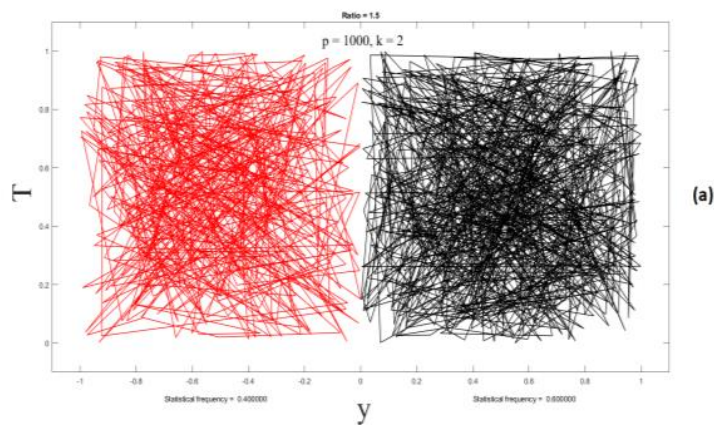

Fig. 5. Results of numerical simulation for asymmetry ef in relative frequency of realization for random event $\Delta y>0$ compared with that of $\Delta y<0$ at fixed $k$. Shown case for volume of random events $p=1000$ and $k=2$. The random events for $\Delta y<0$ are in red, for $\Delta y>0$ in black. Statistically, the frequency for event 
$\Delta y>0$ exceeds that of for event $\Delta y<0$. Numerical simulation was done in the MATLAB environment.

\section{References}

1. Smith J.M. The Problems of Biology (The Problems of Science). Oxford: Oxford University Press, 1986. 144 p.

2. Luisi P.L. Origins of Life. 2014. V. 44. No. 4. P. 335-338.

3. Murazo K.R., Pereto J., Moreno A.A. Origins of Life. 2004. V. 34. No. 3. P. 323-346.

4. Lahav N. Origins of Life. 1985. V. 16. P. 129-149.

5. Moldavanov A. In: Proceedings of the AMSM2017. 2017. P. 258-265.

6. Moldavanov A. International Journal of Geometry. 2018. V. 7. No. 2. P. 66-71.

7. Moldavanov A.V. Topology of Organized Chaos. Moscow: Fizmatkniga, 2020. 66 p.

8. Mora T., Bialek W. Journal of Statistical Physics. 2011. V. 144. No. 2. P. 268-302.

9. Schrodinger E. What is Life. Cambridge: Cambridge University Press, 1944. 31 p.

10. Prudnikov A.I., Bryichkov U.A., Marichev O.I. Integrals and Rows. Moscow: Nauka, 1981. 800 p.

11. de Vries M. The Whole Elephant Revealed: Insights Into the Existence and Operation of Universal Laws and the Golden Ratio. London: John Hunt Publishing, 2012. 403 p.

12. Fleischaker G.R. Origins of Life. 1990. V. 20. No. 2. P. 127.

13. Lenski R., Barrick J., Ofria C. PLoS Biol. 2006. V. 4. P. 2190.

14. Timofeeff-Ressovsky N.W., Zimmer K.G., Delbruck M. Nachr. Ges. Wiss. Göttingen, N.F. 1935. V. 1. No. 13. P. 189-245.

15. England J.L. Nature Nanotechnology. 2015. V. 10. No. 11. P. 919-923. 